\title{
Consumo alimentar e síndrome metabólica em adolescentes
}

\author{
Dietary consumption and metabolic syndrome in adolescents
}

\author{
Maíra Macário de Assis'1 , Juliana Farias de Novaes², Ana Paula Carlos Cândido³ \\ Isabel Cristina Gonçalves Leite ${ }^{1}$, Renata Maria Souza Oliveira ${ }^{3} \bowtie$ \\ ${ }^{1}$ Curso de Mestrado em Saúde Coletiva da Faculdade de Medicina da Universidade Federal de Juiz de Fora. Juiz de Fora, MG. \\ ${ }^{2}$ Departamento de Nutrição e Saúde da Universidade Federal de Viçosa. Viçosa, MG. \\ ${ }^{3}$ Departamento de Nutrição da Universidade Federal de Juiz de Fora. Juiz de Fora, MG
}

\section{RESUMO}

Objetivos: Determinar a prevalência de síndrome metabólica em adolescentes e comparar o consumo alimentar dos indivíduos com e sem a síndrome.

Métodos: Foi conduzido um estudo transversal com adolescentes de 15 a 17 anos de idade, matriculados em escolas públicas e privadas do município de Juiz de Fora, Minas Gerais, Brasil. Utilizou-se o índice de massa corporal para determinar o estado nutricional, classificado de acordo com a Organização Mundial de Saúde. A medida de circunferência da cintura foi aferida segundo Petroski 2003. Para o diagnóstico da síndrome metabólica foram coletados dados de triglicerídeos, colesterol ligado à lipoproteína de alta densidade, glicemia de jejum e pressão arterial e utilizados os critérios da International Diabetes Federation. Para a avaliação dietética utilizou-se um registro alimentar de três dias não consecutivos. Na análise estatística para a associação entre os grupos, foram usados os testes qui quadrado e exato de Fisher e os testes $t$ de Student (teste paramétrico) ou Mann Whitney (não paramétrico).

Resultados: Foram avaliados 302 adolescentes, sendo 51,99\% do sexo masculino e 73,51\% estudantes de escolas públicas A prevalência de obesidade foi de $27,48 \%$ e a de síndrome metabólica de $3,97 \%$, sendo esta de $4,83 \%$ entre as meninas e $3,18 \%$ entre os meninos ( $p=0,46$ ). A prevalência de síndrome metabólica entre os alunos de escolas públicas foi de $4,50 \%$ e entre os de escolas privadas de $2,50 \%$ ( $\mathrm{p}=0,73$ ). $\mathrm{Na}$ comparação entre os grupos com e sem a síndrome, não foram observadas diferenças estatísticas significativas na adequação calórica e de micronutrientes, exceto de vitamina $\mathrm{D}(\mathrm{p}=0,01)$. Verificou-se que os adolescentes com síndrome metabólica consumiam menos vitamina $\mathrm{D}$ do que aqueles sem a síndrome.

Conclusões: A prevalência de síndrome metabólica encontrada foi relevante, considerando-se sua complexidade, gravidade e a faixa etária em estudo. Detectou-se menor ingestão de vitamina D entre os adolescentes com síndrome metabólica.

DESCRITORES: síndrome X metabólica; adolescente; consumo de alimentos; fatores de risco.

\section{ABSTRACT}

Aims: To determine the prevalence of metabolic syndrome in adolescents and to compare the dietary intake of individuals with and without the syndrome.

Methods: A cross-sectional study was conducted with adolescents aged 15 to 17 years, enrolled in public and private schools in Juiz de Fora, Minas Gerais, Brazil. Body mass index was used to determine the nutritional status, classified according to the World Health Organization. Waist circumference was measured according to Petroski (2003). Triglyceride, high-density lipoprotein cholesterol, fasting blood glucose, and blood pressure were assessed and the International Diabetes Federation criteria were used for the diagnosis of metabolic syndrome. A non-consecutive 3-day food record was used for dietary assessment. The chi-square test, Fisher's exact test, Student's parametric $t$ test or the Mann-Whitney $U$ test were used for associations between the groups.

Results: A total of 302 adolescents $-51.99 \%$ males and $73.51 \%$ from public schools - were evaluated. The prevalence of obesity was $27.48 \%$ and that of metabolic syndrome was $3.97 \% ; 4.83 \%$ among girls and $3.18 \%$ among boys $(\mathrm{p}=0.46)$. The prevalence of metabolic syndrome among public school students was $4.50 \%$ compared with $2.50 \%(\mathrm{p}=0.73)$ among those from private schools. In the comparison between the groups with and without metabolic syndrome, there were no statistically significant differences in dietary energy and micronutrient adequacy, except for vitamin $\mathrm{D}(\mathrm{p}=0.01)$. Adolescents with metabolic syndrome consumed less vitamin $\mathrm{D}$ than those without the syndrome.

Conclusions: The prevalence of metabolic syndrome was found to be relevant considering its complexity, severity, and the age group studied. Lower vitamin D intake was observed among adolescents with metabolic syndrome.

KEY WORDS: metabolic syndrome X; adolescent; food consumption; risk factors. 
Abreviaturas: CC, circunferência da cintura; ERICA, Estudo de Riscos Cardiovasculares em Adolescentes; HDL-c, colesterol ligado à lipoproteína de alta densidade; IDF, International Diabetes Federation; IMC, índice de massa corporal; OMS, Organização Mundial de Saúde; PA, pressão arterial; $\mathrm{PAD}$, pressão arterial diastólica; PAS, pressão arterial sistólica; $\mathrm{SM}$, síndrome metabólica; TG, triglicerídeos.

\section{INTRODUÇÃO}

Segundo a Organização Mundial de Saúde (OMS) adolescência é o período que compreende a faixa etária de 10 a 19 anos e que envolve intensas modificações físicas, psíquicas e comportamentais [1]. É uma fase considerada crítica para o início da obesidade, quadro clínico que pode predispor os indivíduos a alterações no metabolismo que incluem a síndrome metabólica (SM) [2].

Trata-se a SM de um transtorno complexo caracterizado por um conjunto de fatores de risco cardiovasculares, como circunferência da cintura (CC), pressão arterial (PA), triglicerídeos (TG) e glicemia elevados, com baixo colesterol ligado à lipoproteína de alta densidade (HDL-c), que contribuem para o desenvolvimento de aterosclerose, dislipidemia, hipertensão arterial e diabetes mellitus tipo II $[3,4]$.

Tanto a obesidade quanto a SM vêm atingindo cada vez mais adolescentes brasileiros, como mostram diferentes pesquisas. De acordo com dados da Pesquisa de Orçamento Familiar de 2008/2009, a prevalência de obesidade foi de 4,9\% [5]. Já na Pesquisa Nacional de Saúde do Escolar (PeNSE) de 2015, que avaliou estudantes de escolas públicas e privadas de 13 a 17 anos de idade, essa prevalência foi para $7,8 \%$, apresentando maiores frequências alunos da região Sul, de escolas privadas e do sexo masculino [6]. Em relação à SM, o Estudo de Riscos Cardiovasculares em Adolescentes (ERICA), que avaliou indivíduos de 12 a 17 anos, registrou prevalência de SM de 2,6\% [7].

Quanto às possíveis causas que podem desencadear a obesidade e a SM estão os fatores genéticos, socioeconômicos, ambientais e de estilo de vida como o tabagismo, a prática de atividade física e os hábitos alimentares [8]. De todas elas, é importante destacar o papel fundamental do consumo alimentar no surgimento de alterações nos fatores de risco envolvidos na SM e também na sua prevenção e controle $[9,10]$. No Brasil, o padrão alimentar dos adolescentes é caracterizado por uma dieta pobre em fibras alimentares, baixo consumo de hortaliças, frutas, leite e feijão e consumo frequente de frituras e produtos ultraprocessados (biscoitos recheados e salgados, guloseimas, bebidas açucaradas e embutidos) ricos em carboidratos refinados, gorduras saturadas, conservantes químicos e sódio [11,12], o que representa um risco, uma vez que estudos têm associado a ingestão de nutrientes específicos como os ácidos graxos saturados e o colesterol com a obesidade e a SM $[13,14]$.

Sabe-se que a adolescência é uma fase importante para adoção de hábitos saudáveis que podem garantir crescimento e desenvolvimento adequados e influenciar muitos aspectos da vida adulta [15]. Por isso, uma alimentação adequada durante esse período é crucial para uma vida saudável e para a prevenção, em longo prazo, de alterações metabólicas e doenças crônicas não transmissíveis [16]. Diante disso, o presente estudo teve como objetivo determinar a prevalência de SM em adolescentes e comparar o consumo alimentar dos indivíduos com e sem SM.

\section{MÉTODOS}

\section{Delineamento e seleção da amostra}

Foi conduzido um estudo transversal com adolescentes de ambos os sexos, com idades entre 15 e 17 anos, matriculados em escolas públicas e privadas do município de Juiz de Fora, Minas Gerais, Brasil. A coleta de dados ocorreu durante o ano de 2012.

Para determinar o tamanho amostral foi considerada uma população total de 11.581 estudantes na faixa etária de interesse. Utilizou-se o cálculo para estudos transversais atendendo aos seguintes critérios: frequência esperada de obesidade de 5\% [5], variabilidade aceitável de $2,5 \%$, nível de confiança de $95 \%$ e $5 \%$ para recuperar possíveis perdas, totalizando 300 indivíduos. O programa utilizado foi o Epi Info versão 6.04.

O município de Juiz de Fora conta com 57 escolas, sendo 36 públicas e 21 privadas. Desse total, 56\% concentram-se na região central $(\mathrm{n}=32)$, sendo 13 públicas e 19 privadas. Participaram do estudo 15 escolas que representam $47 \%$ do total de escolas da região central e $26 \%$ do total de escolas do município. As escolas participantes foram selecionadas com base no cálculo do número de instituições públicas e privadas necessárias para representar a região central.

$$
n=\frac{N_{A} \times N_{B}}{N}
$$

$n=$ número de escolas públicas (ou privadas) a serem avaliadas;

$N_{A}=$ total de escolas públicas (ou privadas) na cidade; $N_{B}=$ total de escolas públicas (ou privadas) na região central da cidade;

$N=$ número total de escolas no município. 
Todos os adolescentes com idade entre 15 e 17 anos foram convidados a participar do estudo. Essa faixa etária foi estabelecida como critério de inclusão com objetivo de homogeneizar a amostra, incluindo adolescentes já com início de maturação sexual e evitando que as diferenças fisiológicas entre as diversas etapas de maturação interferissem nos resultados. Aqueles que aceitaram participar foram submetidos a uma triagem inicial, para determinação do estado nutricional e avaliação dos fatores de inclusão e exclusão. Os critérios de exclusão foram uso crônico ou prolongado de medicamentos como corticoides, anticonvulsivantes e anti-inflamatórios; utilização de suplementos vitamínicos; ser gestante ou lactante; meninas que não haviam menstruado ou haviam tido menarca há menos de um ano antes do momento da coleta de dados; e meninos sem pelos axilares e faciais. A partir da triagem inicial foram selecionados pares de adolescentes, sendo um eutrófico e um com excesso de peso em cada par.

\section{Ética}

O trabalho foi aprovado pelo Comitê de Ética e Pesquisa com Seres Humanos da Universidade Federal de Juiz de Fora (parecer no 066/2009). Todos os avaliados aceitaram voluntariamente participar entregando o termo de consentimento livre e esclarecido assinado pelos pais ou responsáveis.

\section{Avaliação antropométrica}

A partir das medidas de peso e estatura, o índice de massa corporal (IMC) foi calculado utilizando o programa Anthro Plus ${ }^{\circledR}$. O estado nutricional foi determinado considerando-se o escore z para o índice IMC/idade e classificado segundo os critérios propostos pela OMS (2007) [17]. O peso foi obtido em balança portátil digital eletrônica (Tanita ${ }^{\circledR}$, modelo BC 553, Illinois, Estados Unidos), com capacidade máxima de $150 \mathrm{~kg}$, subdivisão em $100 \mathrm{~g}$ e sensibilidade de $50 \mathrm{~g}$. Os adolescentes foram pesados descalços e com o mínimo de roupa [18]. A estatura foi aferida por meio de antropômetro vertical portátil (Alturaexata ${ }^{\circledR}$, Belo Horizonte, Minas Gerais, Brasil), com extensão de $2 \mathrm{~m}$ e escala de $0,1 \mathrm{~cm}$, segundo as normas preconizadas por Jelliffe (1968) [18]. A CC foi aferida na menor circunferência do abdômen, sob roupas e no final de uma expiração normal, utilizando-se uma fita métrica flexível e inelástica [19]. O ponto de corte considerado para essa medida foi o proposto pela International Diabetes Federation
(IDF) indicado para a classificação da SM [20]. Os dados foram coletados por equipe previamente treinada utilizando o espaço físico da própria escola.

\section{Análise dietética}

A análise dietética foi realizada utilizando registro alimentar de três dias não consecutivos, referentes a dois dias de semana e um final de semana. Os registros alimentares foram conferidos utilizando-se álbum fotográfico. Para a análise, receitas e medidas caseiras foram padronizadas por meio de tabelas de composição de alimentos [21,22]. A transformação em gramas foi feita previamente à análise do consumo realizado por meio do programa DietPro ${ }^{\circledR}$ versão 4.0. Foi avaliada a ingestão energética, de proteínas, carboidratos, lipídios, cálcio, vitamina $\mathrm{D}$, fibras, ácidos graxos saturados, ácidos graxos monoinsaturados, ácidos graxos poli-insaturados e colesterol. A ingestão de cálcio foi ajustada pela energia considerando método do nutriente residual [23].

Para o cálculo das necessidades energéticas estimadas (EER) foram utilizadas equações específicas para a faixa etária de estudo, gênero e estado nutricional, as quais foram utilizadas para a adequação de energia [24]. A adequação de cálcio e vitamina $\mathrm{D}$ foi calculada de acordo com a necessidade média estimada [24,25]. O consumo de fibras foi avaliado com base nos valores de ingestão adequada [24].

\section{Análise bioquímica e avaliação da pressão arterial}

A coleta de sangue foi realizada no período da manhã (entre $7 \mathrm{~h}$ e $8 \mathrm{~h} 30$ ) por profissionais capacitados do Hospital Universitário da Universidade Federal de Juiz de Fora, na própria escola em ambiente previamente preparado. Os adolescentes foram submetidos à punção venosa, após jejum de 12 horas, para obtenção das amostras sanguíneas. Foram analisados TG, HDL-c e glicemia de jejum.

A PA foi aferida com o indivíduo sentado, através de equipamento automático, usando manguito de tamanho apropriado. Foram realizadas três medidas, em intervalos de cinco minutos, sendo adotada a média das duas últimas aferições. As orientações seguidas foram da VI Diretrizes Brasileiras de Hipertensão Arterial [26]. Os pontos de corte considerados foram os propostos pela IDF indicados para a classificação da SM [20]. 


\section{Classificação da síndrome metabólica}

Utilizou-se para classificação da SM o critério da IDF [20], que estabelece para o diagnóstico a presença de pelo menos três dos seguintes componentes alterados, sendo obrigatório a CC elevada: HDL-c, TG, glicemia de jejum, pressão arterial sistólica (PAS) e pressão arterial diastólica (PAD). Os pontos de corte considerados estão relacionados no Quadro 1.

Quadro 1. Pontos de corte considerados para classificação de síndrome metabólica em adolescentes Fonte: Zimmet et al., 2007 [20].

\begin{tabular}{|l|l|}
\hline Circunferência da cintura & $\begin{array}{l}<16 \text { anos: Percentil } 90 \\
\geq 16 \text { anos, sexo masculino: } \geq 90 \mathrm{~cm} \\
\geq 16 \text { anos, sexo feminino: } \geq 80 \mathrm{~cm}\end{array}$ \\
\hline $\begin{array}{l}\text { HDL-c (colesterol ligado } \\
\text { à lipoproteína de alta } \\
\text { densidade) }\end{array}$ & $\begin{array}{l}<16 \text { anos: } 40 \mathrm{mg} / \mathrm{dL} \\
\geq 16 \text { anos, sexo masculino: }<40 \mathrm{mg} / \mathrm{dL} \\
\geq 16 \text { anos, sexo feminino: }<50 \mathrm{mg} / \mathrm{dL}\end{array}$ \\
\hline Triglicerídeos & $\geq 150 \mathrm{mg} / \mathrm{dL}$ \\
\hline Glicemia de jejum & $\geq 100 \mathrm{mg} / \mathrm{dL}$ \\
\hline Pressão arterial sistólica & $\geq 130 \mathrm{mmHg}$ \\
\hline Pressão arterial diastólica & $\geq 85 \mathrm{mmHg}$ \\
\hline
\end{tabular}

\section{Análise estatística}

A descrição das variáveis foi feita a partir da determinação das medidas de tendência central e dispersão. Para verificar a distribuição das variáveis utilizou-se o teste Shapiro-Wilk. Para avaliar a associação entre as variáveis qualitativas foi aplicado o qui quadrado ou o teste exato de Fisher (em caso de caselas menores que 5). A comparação entre as variáveis quantitativas foi feita utilizando os testes $t$ de Student (paramétricas) e Mann-Whitney (não paramétricas) conforme distribuição das variáveis. Adotou-se nível de significância estatística de 5\%. A análise dos dados foi realizada utilizando o programa STATA versão 13.0.

\section{RESULTADOS}

Por meio do levantamento do diário de classe foram encontrados 4.357 indivíduos nas escolas da região central, dentro da faixa etária determinada como critério de inclusão (15 a 17 anos); destes, 2.524 alunos aceitaram participar e foram submetidos à triagem inicial, conforme descrito na metodologia. A partir da triagem inicial foram selecionados 152 adolescentes eutróficos e 152 com excesso de peso. Dois adolescentes declinaram posteriormente da participação, porque iniciaram acompanhamento nutricional com outros profissionais, sendo a amostra final constituída por 302 adolescentes, dos quais 157 $(51,99 \%)$ eram do sexo masculino e $222(73,51 \%)$ estudavam em escolas públicas. A média de idade foi de $16,03 \pm 0,78$ e a mediana de 16 anos. Em relação aos dados antropométricos, foram encontrados valores mé-

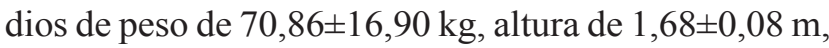
CC de 77,10 $\pm 11,64 \mathrm{~cm}$ e IMC de $24,75 \pm 5,30 \mathrm{~kg} / \mathrm{m}^{2}$.

A prevalência de excesso de peso foi de 50,33\% $(\mathrm{n}=152)$ e de obesidade $27,48 \%(\mathrm{n}=83)$. A prevalência de SM foi de 3,97\% ( $n=12)$, sendo 4,83\% $(n=7)$ entre o sexo feminino e $3,18 \%(n=5)$ entre o sexo masculino $(\mathrm{p}=0,46)$. A prevalência de síndrome metabólica entre os alunos de escolas públicas foi de 4,50\% $(\mathrm{n}=10)$ e entre os de escolas privadas de $2,50 \%(n=2)(p=0,73)$.

A Tabela 1 descreve a prevalência dos componentes da $\mathrm{SM}$ em todos os avaliados e nos classificados com e sem SM. Os maiores percentuais de alteração no total dos participantes foram de HDL-c baixo $(23,51 \%)$ e CC elevada $(19,87 \%)$ sendo essas prevalências de $100 \%$ nos adolescentes com SM. Em relação aos TG e à PA elevados, apesar de apresentarem menores prevalências quando comparados aos demais componentes, estiveram alterados em mais da metade daqueles com SM. A prevalência de alteração da glicemia na amostra total foi a mais baixa $(2,90 \%)$, o

Tabela 1. Prevalências dos componentes da síndrome metabólica entre adolescentes de 15 a 17 anos. Juiz de Fora, Minas Gerais, 2012.

\begin{tabular}{|c|c|c|c|c|}
\hline \multirow{2}{*}{ Componentes da SM } & \multicolumn{2}{|c|}{ Total $(n=302)$} & \multirow{2}{*}{$\begin{array}{c}\text { Com SM } \\
\text { n (\%) }\end{array}$} & \multirow{2}{*}{$\begin{array}{c}\text { Sem SM } \\
\text { n (\%) }\end{array}$} \\
\hline & n (\%) & IC 95\% & & \\
\hline CC elevada & $60(19,87)$ & $15,52-24,82$ & $12(100,00)$ & $48(16,55)$ \\
\hline Obesidade pelo IMC & $83(27,48)$ & $17,41-37,33$ & $12(100,00)$ & $71(24,48)$ \\
\hline HDL-c baixo & $71(23,51)$ & $18,84-28,70$ & $12(100,00)$ & $59(20,34)$ \\
\hline TG elevados & $24(7,95)$ & $5,16-11,59$ & $8(66,66)$ & $16(5,52)$ \\
\hline Glicemia elevada & $9(2,90)$ & $1,37-5,58$ & $0(0,00)$ & $9(3,10)$ \\
\hline PA elevada & $23(7,62)$ & $4,89-11,21$ & $7(58,33)$ & $16(5,52)$ \\
\hline
\end{tabular}

SM, síndrome metabólica; CC, circunferência da cintura; IMC, índice de massa corporal; HDL-C, colesterol ligado a lipoproteínas de alta densidade; TC, triglicerídeos; PA, pressão arterial; IC95\%, intervalo de confiança de 95\%. 
que foi observado também entre os adolescentes com SM, pois em nenhum deles foi verificada glicemia elevada (Tabela 1).

Em relação ao consumo alimentar, a Tabela 2 mostra o consumo mediano de energia, macro e micronutrientes dos adolescentes, estratificados por presença da SM. Somente a ingestão de vitamina $\mathrm{D}$ foi diferente entre os grupos, sendo menor em adolescentes com $\mathrm{SM}(\mathrm{p}=0,01)$.

A Tabela 3 apresenta a comparação da adequação calórica e de micronutrientes dos adolescentes avaliados estratificada por presença de SM, obtida pela média do registro alimentar de três dias. Não foram observadas diferenças estatísticas significativas entre os grupos.

\section{DISCUSSÃO}

A prevalência de SM entre os adolescentes avaliados foi elevada $(3,97 \%)$ quando comparada aos dados nacionais do ERICA $(2,6 \%)$ [7], ambos utilizando o critério diagnóstico da IDF [20]. Para a identificação da SM existem outras referências propostas como a de Cook et al. [27] e a de De Ferranti et al. [28] baseadas no National Cholesterol Education Program. Dependendo do critério escolhido, a prevalência da SM pode variar, uma vez que eles diferem em relação aos componentes e pontos de corte utilizados. Costa et al. [29] demonstraram isso, ao comparar a proporção de adolescentes com SM utilizando os três diferentes critérios citados anteriormente em uma mesma amostra.

Tabela 2. Consumo mediano de energia, macro e micronutrientes dos adolescentes avaliados (faixa etária entre 15 e 17 anos), estratificado por presença da síndrome metabólica. Juiz de Fora, Minas Gerais, 2012.

\begin{tabular}{|c|c|c|c|c|c|}
\hline \multirow{2}{*}{ Variáveis } & \multicolumn{2}{|c|}{ Com SM } & \multicolumn{2}{|c|}{ Sem SM } & \multirow{2}{*}{$\mathbf{p}^{*}$} \\
\hline & Mediana & IIQ & Mediana & IIQ & \\
\hline Energia (kcal) & 2087,26 & $2486,54-1564,34$ & 2193,33 & $2596,32-1776,56$ & 0,57 \\
\hline Proteínas (g) & 56,86 & $93,62-48,53$ & 73,72 & $95,83-59,76$ & 0,32 \\
\hline Carboidratos (g) & 273,38 & $311,54-222,80$ & 288,86 & $341,85-232,24$ & 0,61 \\
\hline Lipídios (g) & 68,98 & $95,12-61,81$ & 79,19 & $96,09-63,96$ & 0,40 \\
\hline Cálcio (mg) & 417,69 & $561,78-325,10$ & 551,71 & $707,52-379,53$ & 0,08 \\
\hline Vitamina D (mcg) & 0,96 & $1,66-0,53$ & 2,33 & $3,65-1,36$ & 0,01 \\
\hline Fibras (g) & 11,67 & $14,64-8,56$ & 11,53 & $15,15-8,54$ & 0,91 \\
\hline AGMI (g) & 16,74 & $29,08-12,97$ & 19,23 & $25,50-14,10$ & 0,81 \\
\hline AGPI (g) & 18,75 & $28,49-15,16$ & 16,41 & $22,24-12,77$ & 0,27 \\
\hline AGS (g) & 15,10 & $23,88-12,71$ & 19,67 & $23,91-13,65$ & 0,53 \\
\hline Colesterol (g) & 159,94 & $303,17-122,54$ & 200,41 & $269,25-137,74$ & 0,75 \\
\hline
\end{tabular}

AGMI, ácidos graxos monoinsaturados; AGPI, ácidos graxos poli-insaturados; AGS, ácidos graxos saturados; IIQ, intervalo interquartil (percentil 75-percentil 25).

* Teste de Mann-Whitney.

Tabela 3. Comparação da adequação de ingestão calórica e de micronutrientes nos adolescentes de 15 a 17 anos, estratificada por presença da síndrome metabólica. Juiz de Fora, Minas Gerais, 2012.

\begin{tabular}{|c|c|c|c|c|}
\hline Variáveis & $\begin{array}{l}\text { Total } \\
\text { n (\%) }\end{array}$ & $\begin{array}{c}\text { Com SM } \\
\text { n (\%) }\end{array}$ & $\begin{array}{c}\text { Sem SM } \\
\text { n (\%) }\end{array}$ & $\mathbf{p}^{*}$ \\
\hline \multicolumn{5}{|l|}{ Consumo calórico } \\
\hline Acima da necessidade energética estimada & $104(34,46)$ & $2(0,66)$ & $102(33,80)$ & \multirow[t]{2}{*}{0,19} \\
\hline Abaixo da necessidade energética estimada & $198(65,54)$ & $10(3,31)$ & $188(62,25)$ & \\
\hline \multicolumn{5}{|l|}{ Consumo de cálcio } \\
\hline Adequado & $34(11,26)$ & $2(0,66)$ & $32(10,60)$ & \multirow[t]{2}{*}{0,55} \\
\hline Inadequado & $268(88,74)$ & $10(3,32)$ & $258(85,43)$ & \\
\hline \multicolumn{5}{|l|}{ Consumo de vitamina D } \\
\hline Adequado & $1(0,63)$ & $0(0,00)$ & $1(0,63)$ & \multirow[t]{2}{*}{0,84} \\
\hline Inadequado & $159(99,37)$ & $6(3,75)$ & $153(95,62)$ & \\
\hline \multicolumn{5}{|l|}{ Fibras } \\
\hline Acima da ingestão adequada & $31(10,26)$ & $2(0,66)$ & $29(9,60)$ & \multirow[t]{2}{*}{0,50} \\
\hline Abaixo da ingestão adequada & $271(89,74)$ & $10(3,31)$ & $261(86,43)$ & \\
\hline
\end{tabular}

* Teste exato de Fisher. 
A maior proporção foi verificada utilizando a proposta de De Ferranti et al. [28], seguida de Cook et al. [27] e da IDF [20]. Para os autores, as maiores prevalências encontradas por De Ferranti podem ser explicadas, justamente, por seus valores de corte menos rigorosos para perímetro abdominal, HDL-c e TG [28].

A ausência de um critério diagnóstico estabelecido e de pontos de corte considerados de risco para o público adolescente acarreta ampla variação de prevalência entre os estudos [10]. No Brasil, dependendo do critério adotado ela pode variar de $37 \%$ em Goiás, $16,6 \%$ em Viçosa a $6,7 \%$ no Paraná e $1,32 \%$ em Vitória [30,31]. A padronização desses aspectos facilitaria a detecção precoce da SM e permitiria uma comparação mais eficaz entre pesquisas realizadas em diferentes localidades.

Observou-se no presente estudo uma diferença no percentual de estudantes com SM em escolas públicas em relação aos de escolas privadas, porém esta não foi estatisticamente significativa. No estudo do ERICA, verificou-se uma maior prevalência de SM nos estudantes de escolas públicas, o que pode indicar uma possível associação do nível socioeconômico com a SM [7]. Em Montes Claros, Minas Gerais, Cruz et al. [32], ao avaliarem 382 estudantes de 10 a 16 anos, encontraram maior prevalência de SM $(8,70 \%)$ entre os adolescentes de classe socioeconômica mais baixa. No entanto, quanto ao estado nutricional, estudos brasileiros verificaram a existência de uma associação entre o maior nível socioeconômico e a presença de excesso de peso $[33,34]$.

Com relação ao consumo mediano de energia, macro e micronutrientes, observou-se menor ingestão de vitamina D entre os adolescentes com SM. Dentre as fontes alimentares dessa vitamina estão a gema de ovo, o fígado e a manteiga. Entre os alimentos ricos estão o salmão, o atum e o óleo de fígado de bacalhau, porém esses alimentos ou não são consumidos em grandes quantidades, ou não fazem parte da alimentação do brasileiro [35]. Dentre os poucos alimentos fonte que fazem parte do hábito alimentar da população brasileira está o leite [22]. Porém, o café da manhã, refeição na qual há maior consumo de produtos lácteos, é considerada a mais omitida pelos adolescentes [36]. Outra fonte importante de vitamina $\mathrm{D}$ é a formação endógena desencadeada pela exposição à luz solar [37].

Tanto a ingestão dietética quanto a exposição solar interferem na concentração sérica da forma circulante da vitamina D, o 25-hidroxicolecalciferol [25 (OH) D] [38]. Nos Estados Unidos, um estudo que investigou o consumo e os níveis de vitamina D em 145 crianças e adolescentes de nove a 15 anos encontrou que a maioria dos avaliados $(83,4 \%)$ apresentavam deficiência de vitamina $\mathrm{D}$, sendo que a ingestão da vitamina teve relação com os seus níveis séricos [39].

Além da já conhecida importância da vitamina D para a saúde óssea, o comprometimento no nível sérico também tem sido apontado como possível fator predisponente a alterações metabólicas e doenças cardiovasculares, como a obesidade e a hipertensão arterial [40]. Essa é uma possível explicação para o achado do presente estudo de que os adolescentes com $\mathrm{SM}$ consumiam menos vitamina $\mathrm{D}$ do que aqueles sem SM.

Oliveira et al. [41], avaliando adolescentes brasileiros de 15 a 17 anos, encontraram menor ingestão de vitamina $\mathrm{D}$ entre os indivíduos com excesso de peso quando comparados com os eutróficos. Além disso, foram observados menores níveis séricos de $25(\mathrm{OH}) \mathrm{D}$ entre aqueles com excesso de peso, obesidade abdominal e hipercolesterolemia [41]. Essa relação pode ser justificada pelo depósito de $25(\mathrm{OH})$ $\mathrm{D}$ nos adipócitos, diminuindo sua biodisponibilidade e desencadeando uma cascata de reações hormonais que resultam em aumento da fome e diminuição do gasto energético [42]. Em adolescentes obesos, baixas concentrações de vitamina $\mathrm{D}$ também têm sido associadas com níveis reduzidos de HDL-c [43] e elevados de triglicerídeos e do índice homeostatic model assessment para resistência à insulina (HOMA-IR) [44]. Um estudo realizado com 3.577 adolescentes americanos com idades entre 12 e 19 anos encontrou maior chance de hipertensão arterial e SM para os indivíduos no menor quartil do nível sérico de $25(\mathrm{OH}) \mathrm{D}$, em comparação com o maior quartil, independentemente da adiposidade corporal [45].

Ressalta-se que mesmo os adolescentes sem SM apresentavam consumo inadequado de vitamina $\mathrm{D}$, motivo pelo qual a diferença significativa que aparece na Tabela 2 não aparece na Tabela 3. Peters et al. [46], ao avaliarem 136 adolescentes de 16 a 20 anos em São Paulo, observaram que somente $14,9 \%$ dos avaliados alcançaram a recomendação diária de ingestão adequada da vitamina D. A insuficiência sérica foi observada em $62,1 \%$ dos adolescentes avaliados, prevalência considerada alta, uma vez que o Brasil apresenta clima ensolarado. Os autores atribuíram a taxa elevada de deficiência de vitamina $\mathrm{D}$ à baixa ingestão [46].

Não foram observadas diferenças significativas entre os grupos quanto ao percentual de adequação do consumo alimentar. Resultado semelhante foi encontrado entre 100 meninas de 14 a 17 anos, com e sem SM, avaliadas em Viçosa, Minas Gerais [15]. 
Considerando que, por meio da análise quantitativa, não foi possível identificar diferenças entre os grupos, como alternativa sugere-se avaliar a qualidade da dieta buscando marcadores e padrões de consumo inadequados entre os indivíduos, que possam estar relacionadas ao desenvolvimento da SM $[4,47]$.

O presente estudo teve como limitações questões relativas aos estudos de consumo, como a dificuldade em mensurar a exposição, devido à complexidade da dieta e à subnotificação da ingestão alimentar. Além disso, em razão do delineamento transversal não foi possível estabelecer relações causais entre a associação encontrada relativa ao consumo de vitamina $D$ e à presença de SM. Por fim, sugere-se que estudos futuros investiguem a vitamina D e o seu papel na SM em adolescentes, analisando não somente a ingestão, mas também a dosagem sérica da $25(\mathrm{OH}) \mathrm{D}$.

Concluindo, a prevalência de SM observada foi relevante, considerando-se a faixa etária em estudo, a complexidade e a gravidade da síndrome. Ademais, foi detectada menor ingestão de vitamina D entre os adolescentes com SM. Sugere-se a necessidade de se estimular o consumo de alimentos fonte dessa vitamina, não somente por sua relação com a saúde óssea, mas também por sua possível ação protetora a alterações metabólicas e doenças cardiovasculares.

\section{NOTAS}

\section{Apoio financeiro}

Este estudo recebeu apoio financeiro da Fundação de Amparo à Pesquisa de Minas Gerais (FAPEMIG), processo $\mathrm{n}^{\mathrm{o}}$ APQ 01571.

\section{Declaração de conflito de interesses}

As autoras declaram não haver conflitos de interesses relevantes ao conteúdo deste estudo, informam ter tido acesso a todos os dados obtidos e assumem completa responsabilidade pela integridade dos resultados.

\section{REFERÊNCIAS}

1. Priore SE, Faria FR, Franceschini SCC. Adolescência. In: Priore SE, Oliveira RMS, Faria ER, Franceschini SCC, Pereira PF, Organizadores. Nutrição e saúde na adolescência. Rio de Janeiro: Editora Rubio; 2010. p. 1-4.

2. World Health Organization. Nutrition in adolescence - Issues and Challenges for the Health Sector: Issues in Adolescent Health and Development [Internet]. Geneva: WHO; 2005 [cited 2016 July 01]. Available from: http://apps.who.int/iris/ bitstream/10665/43342/1/9241593660_eng.pdf

3. Sociedade Brasileira de Cardiologia, Organizador. I Diretriz Brasileira de Diagnóstico e Tratamento da Síndrome Metabólica [Internet]. 2005 [cited 2016 July 01]. Available from: http://publicacoes.cardiol.br/consenso/2005/sindromemetabolica.pdf

4. Bressan J, Hermsdorff HHM, Zulet M, Martnez JA. Impacto hormonal e inflamatório de diferentes composições dietéticas: ênfase em padrões alimentares e fatores dietéticos específicos. Arq Bras Endocrinol Metab. 2009;53(5):572-81. https:// doi.org/10.1590/S0004-27302009000500010

5. Instituto Brasileiro de Geografia e Estatística. Pesquisa de Orçamentos Familiares 2008-2009: Antropometria e estado nutricional de crianças, adolescentes e adultos no Brasil. Rio de Janeiro: IBGE; 2010.

6. Instituto Brasileiro de Geografia e Estatística. Pesquisa Nacional de Saúde do Escolar: 2015. Rio de Janeiro: IBGE; 2016.

7. Kuschnir MCC, Bloch KV, Szklo M, Klein CH, Barufaldi LA, Abreu GA, Schaan B, Veiga GV, Silva TLN, Vasconcellos MTL, Moraes AJP, Oliveira AMA, Tavares BM, Oliveira CL, Cunha CF, Giannini DT, Belfort DR, Santos EL, Leon EB, Oliveira ERA, Fujimori E, Borges AL, Magliano ES, Assis F, Vasconcelos G, Azevedo GD, Brunken GS, Guimarães ICB, Faria Neto JR, Oliveira JS, Carvalho KMB, Gonçalves LGO, Monteiro MI, Santos MM, Muniz PT, Jardim PCBV, Ferreira PAM, Montenegro Jr RM, Gurgel RQ. ERICA: prevalence of metabolic syndrome in Brazilian adolescents. Rev Saúde Pública. 2016;50:1s-13s. https://doi.org/10.1590/s01518-8787.2016050006701

8. Expert Panel on Integrated Guidelines for Cardiovascular Health and Risk Reduction in Children and Adolescents: Summary Report. Pediatrics [Internet]. 2011 [cited 2016 July 01]. Available from: http://pediatrics.aappublications.org/ content/128/Supplement_5/S213.full.pdf+html

9. Perez GH, Romano BW. Comportamento Alimentar e síndrome metabólica: aspectos psicológicos. Rev Soc Cardiol Estado de São Paulo. 2004;14(4):544-50.

10. Tavares LF, Yokoo EM, Rosa MLG, Fonseca SC. Síndrome metabólica em crianças e adolescentes brasileiros: revisão sistemática. Cad Saúde Colet. 2010;18(4):469-76.

11. Instituto Brasileiro de Geografia e Estatística. Pesquisa Nacional de Saúde do Escolar 2012. Rio de Janeiro: IBGE; 2013.

12. Pan American Health Organization. Ultra-processed food and drink products in Latin America: Trends, impact on obesity, policy implications. Washington, DC: PAHO; 2015.

13. Traebert J, Moreira EAM, Bosco VL, Almeida ICS. Transição alimentar: problema comum à obesidade e à cárie dentária. Rev Nutr. 2004;17(2):247-53. https://doi.org/10.1590/S1415-52732004000200011

14. Tavares LF, Castro IRR, Levy RB, Cardoso LO, Claro RM. Dietary patterns of Brazilian adolescents: results of the Brazilian National School-Based Health Survey (PeNSE). Cad Saúde Pública. 2014;30(12):2679-90. https://doi. org/10.1590/0102-311X00016814 
15. Faria ER, Faria FR, Pinto CA, Franceschini SCC, Peluzio MCG, Priore SE. Consumo alimentar e síndrome metabólica em adolescentes do sexo feminino. RASBRAN. 2014;6(1):21-8.

16. World Health Organization. Fifty-seven World Health Assembly. Global Strategy on Diet, Physical Activity and Health [Internet]. 2004 [cited 2016 July 01]. Available from: http://www.who.int/dietphysicalactivity/strategy/eb11344/strategy_ english_web.pdf

17. De Onis M, Onyango AW, Borghi E, Siyam A, Nishida C, Siekmann J. Development of a WHO growth reference for school-aged children and adolescents. Bull World Health Organ. 2007 Sept;85(9):660-7. https://doi.org/10.2471/ BLT.07.043497

18. Jelliffe DB. Evaluación del estado de nutrición de la comunidade. Genebra: OMS; 1968.

19. Petroski EL. Antropometria: técnicas e padronizações. Porto Alegre: Palotti; 2003.

20. Zimmet P, Alberti G, Kaufman F, Tajima N, Silink M, Arslanian S, Wong G, Bennett P, Shaw J, Caprio S. The metabolic syndrome in children and adolescents: the IDF consensus. Diab Voice. 2007;52(4):29-32. https://doi.org/10.1111/j.13995448.2007.00271.x

21. Philippi ST. Tabela de composição de alimentos: suporte para decisão nutricional. 2ạ ed. Brasília: Anvisa; 2002.

22. Pinheiro ABV, Lacerda SEM, Benzecry EH. Tabela para avaliação de consumo alimentar em medidas caseiras. 4 $\underline{\text { a }}$ ed. São Paulo: Atheneu; 2002.

23. Willett W, Stampfer M. Implications of total energy intake for epidemiologic analyses. In: Willett W. Nutritional epidemiology. 2a ed. New York: Oxford University Press; 1998. p. 514. https://doi.org/10.1093/acprof:oso/9780195122978.003.11

24. Institute of Medicine. Food and Nutrition Board. Dietary Reference Intakes. Energy, carbohydrate, fiber, fat, fatty acids, cholesterol, protein, and amino acids (macronutrients). Washington, DC: National Academy Press; 2005.

25. Institute of Medicine. Food and Nutrition Board. Dietary Reference Intakes for Calcium and Vitamin D. Washington, DC: National Academy Press; 2010.

26. Sociedade Brasileira de Cardiologia; Sociedade Brasileira de Hipertensão; Sociedade Brasileira de Nefrologia. VI Diretrizes Brasileiras de Hipertensão Arterial. Arq Bras Cardiol. 2010;95(1 Supl 1): 1-51.

27. Cook S, Weitzman M, Auinger P, Nguyen M, Dietz WH. Prevalence of a metabolic syndrome phenotype in adolescents: findings from the third National Health and Nutrition Examination Survey, 1988-1994. Arch Pediatr Adolesc Med. 2003 Aug;157(8):821-7. https://doi.org/10.1001/archpedi.157.8.821

28. De Ferranti SD, Gauvreau K, Ludwing DS, Neufeld EJ, Newburger JW, Rifai N. Prevalence of the metabolic syndrome in American adolescents: findings from the Third National Health and Nutrition Examination Survey. Circulation. 2004;110:2494-7. https://doi.org/10.1161/01.CIR.0000145117.40114.C7

29. Costa RF, Santos NS, Goldraich NP, Barski TF, de Andrade KS, Kruel LF. Metabolic syndrome in obese adolescents: a comparison of three different diagnostic criteria. J Pediatr (Rio J). 2012;88(4):303-9. https://doi.org/10.2223/jped.2200

30. Rodrigues AN, Perez AJ, Pires JGP, Carletti L, Araújo MTM, Moyses MR, Bissoli NS, \& Abreu GR. Fatores de risco cardiovasculares, suas associações e presença de síndrome metabólica em adolescentes. J Pediatr (Rio J). 2009;85(1): 55-60. https://doi.org/10.2223/JPED.1867

31. Neto AS, Bozza R, Ulbrich A, Mascarenhas LPG, Boguszewski MCS, Campos W. Síndrome metabólica em adolescentes de diferentes estados nutricionais. Arq Bras Endocrinol Metab. 2012;56(2):104-9.

32. Cruz IRD, Freitas DA, Soares WD, Mourão DM, Aidar FA, Carneiro AL. Síndrome metabólica e associação com nível socioeconômico em escolares. Rev CEFAC. 2014;16(4):1294-302. https://doi.org/10.1590/1982-021620146713

33. Paula FAR, Lamboglia CMGF, Silva VTBL, Monteiro MS, Moreira AP, Pinheiro MHNP, Silva CAB. Prevalência de sobrepeso e obesidade em escolares da rede pública e particular da cidade de Fortaleza. Rev Bras Promoc Saúde, 2015;27(4):455-61. https://doi.org/10.5020/18061230.2014.p455

34. Miranda JMQ, Palmeira MV, Polito LFT, Brandão MRF, Bocalini DS, Figueira J et AL. Prevalência de sobrepeso e obesidade infantil em instituições de ensino: públicas vs. privadas. Rev Bras Med Esporte. 2015:21(2):104-7. https:// doi.org/10.1590/1517-869220152102143660

35. Calvo MS, Whiting SJ. Public health strategies to overcome barriers to optimal vitamin D status in populations with special needs. J Nutr. 2006;136(4):1135-9.

36. Marchioni DML, Gorgulho BM, Teixeira JA, Junior EV, Fisberg RM. Prevalência de omissão do café da manhã e seus fatores associados em adolescentes de São Paulo: estudo ISA-Capital. Nutrire. 2015;40(1):10-20. https://doi. org/10.4322/2316-7874.032414

37. Bringhurst FR, Demay MB, Kronenberg HM. Hormones and disorders of mineral metabolism. In: Kronenberg HM, Melmed S, Polonsky KS, Larsen PR editors. Williams Textbook of Endocrinology, 11 a ed. Philadelphia: Elsevier; 2008.

38. Strange RC, Shipman KE, Ramachandran S. Metabolic syndrome: A review of the role of vitamin D in mediating susceptibility and outcome. World J Diabetes. 2015 July 10;6(7):896-911. https://doi.org/10.4239/wjd.v6.i7.896

39. Au LE, Economos CD, Goodman E, Must A, Chomitz VR, Sacheck JM. Vitamin D intake and serum vitamin D in ethnically diverse urban schoolchildren. Public Health Nutr. 2012 Nov;15(11):2047-53. https://doi.org/10.1017/ S1368980012003217

40. Leao ALM, Santos LC. Consumo de micronutrientes e excesso de peso: existe relação? Rev Bras Epidemiol. 2012;15(1): 85-95. https://doi.org/10.1590/S1415-790X2012000100008 
41. Oliveira RMS, Novaes JF, Azeredo LM, Cândido APC, Leite ICG. Association of vitamin D insufficiency with adiposity and metabolic disorders in brazilian adolescents. Public Health Nutr. 2014 Apr;17(4):787-94. https://doi.org/10.1017/ S1368980013001225

42. Schuch NJ, Garcia VC, Martini LA. Vitamina D e doenças endocrinometabólicas: [revisão]. Arq Bras Endocrinol Metab. 2009;53(5):625-33. https://doi.org/10.1590/S0004-27302009000500015

43. Smotkin-Tangorra M, Purushothaman R, Gupta A, Nejati G, Anhalt H, Ten S. Prevalence of vitamin D insufficiency in obese children and adolescents. J Pediatr Endocrinol Metab. 2007;20(7):817-23. https://doi.org/10.1515/JPEM.2007.20.7.817

44. Torun E, Gönüllü E, Özgen IT, Cindemir E, Öktem F. Vitamin D Deficiency and Insufficiency in Obese Children and Adolescents and Its Relationship with Insulin Resistance. Int J Endocrinol. 2013;2013:631845. https://doi. org/10.1155/2013/631845

45. Reis JP, von Mühlen D, Miller ER 3rd, Michos ED, Appel LJ. Vitamin D status and cardiometabolic risk factors in the United States adolescent population. Pediatrics. 2009;124(3):e371-9. https://doi.org/10.1542/peds.2009-0213

46. Peters BSE, Santos LC, Fisberg M, Wood RC, Martini LA. Prevalence of vitamin D insufficiency in brazilian adolescents. Ann Nutr Metab. 2009;54:15-21. https://doi.org/10.1159/000199454

47. Hu FB. Dietary pattern analysis a new direction in nutritional epidemiology. Curr Opin Lipidol. 2002;13(1):3-9. https:// doi.org/10.1097/00041433-200202000-00002 